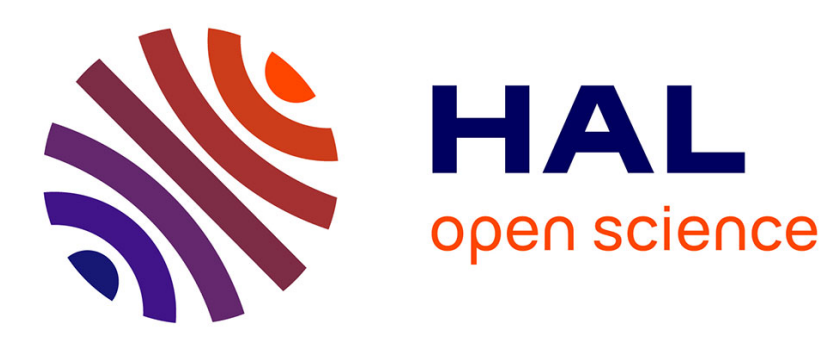

\title{
Essai de désinfection solaire des sols dans le Sud- Est de la France
}

Eric Berninger, Jean-Claude Pionnat, Claude Scotto La Massese

\section{To cite this version:}

Eric Berninger, Jean-Claude Pionnat, Claude Scotto La Massese. Essai de désinfection solaire des sols dans le Sud- Est de la France. Agronomie, 1985, 5 (6), pp.505-513. hal-00884778

\section{HAL Id: hal-00884778 \\ https://hal.science/hal-00884778}

Submitted on 1 Jan 1985

HAL is a multi-disciplinary open access archive for the deposit and dissemination of scientific research documents, whether they are published or not. The documents may come from teaching and research institutions in France or abroad, or from public or private research centers.
L'archive ouverte pluridisciplinaire HAL, est destinée au dépôt et à la diffusion de documents scientifiques de niveau recherche, publiés ou non, émanant des établissements d'enseignement et de recherche français ou étrangers, des laboratoires publics ou privés. 


\title{
Essai de désinfection solaire des sols dans le Sud- Est de la France
}

\author{
Eric BERNINGER, Jean-Claude PIONNAT (*) \& Claude SCOTTO LA MASSESE (**) \\ I.N.R.A., Station d'Amélioration des Plantes Florales, « La Gaudine », F 83600 Fréjus \\ (*) I.N.R.A., Station de Pathologie Végétale, 62 bd du Cap, F 06602 Antibes \\ (**) I.N.R.A., Station de Recherches sur les Nématodes, 123 bd F. Meilland, F 06602 Antibes
}

RÉSUMÉ

\begin{abstract}
L'effet de paillages plastiques continus avec trois types de films (polyéthylène simple, éthylvinylacétate, polyéthylène alvéolé " Bulle ») sur l'échauffement du sol a été mesuré en serres et en plein air dans le Sud-Est de la France. Dans les meilleurs cas, on atteint en été des températures comparables à celles réalisées dans des pays subtropicaux où l'effet de ces techniques sur quelques agents pathogènes a été démontré. L'action sur la microflore et la nématofaune globales du paillage le plus performant a été analysée. Des modifications sensibles et durables des effectifs et une diminution de la diversité des genres et des espèces, variable selon les organismes considérés, ont été obtenues. Elles paraissent plus nettes dans les sols non soumis aux désinfections artificielles et peuvent représenter une forme de désinfection sélective des milieux de culture.
\end{abstract}

Mots clés additionnels : Recolonisation des sols, bactéries, cryptogames, groupes trophiques, « plastiques »

\section{Soil solarization trials in Southeastern France}

The effect of three types of plastic - film mulching (plain polythene, ethylvinylacetate, polythene with air bubbles) on soil heating was measured in summer, outdoors and in glasshouses. The best mulching resulted in temperatures like those obtained in subtropical countries where the control of soil - borne pathogens has been demonstrated.

The effect of the best mulching treatment on the soil microflora and nematofauna showed marked and lasting changes in the number and the diversity of genera and species, which appeared more clearly in soils previously not artificially disinfested. A selective type of soil disinfestation can be achieved.

Additional key words : Soil recolonization, bacteria, fungi, trophic groups, plastics.

\section{INTRODUCTION}

Les traitements thermiques ont été les premiers et sont toujours utilisés en agriculture pour la désinfection des organes de multiplication et des sols. Moins employés par suite de l'accroissement des coûts de l'énergie, leur intérêt, par rapport aux traitements chimiques, subsiste en raison notamment de l'absence de résidus libérés dans les milieux.

La chaleur solaire est d'ailleurs à l'origine de la destruction naturelle de nombreux ravageurs telluriques dans les pays chauds et dans les serres en période estivale. Ainsi, DeMEure (1978) observe que les nématodes du genre Meloidogyne sont tués, au cours de la saison sèche, dans les 20 premiers centimètres des parcelles contaminées du Sahel sénégalais.

L'emploi du paillage plastique pour accroître l'échauffement du sol en vue d'éliminer les ravageurs a été envisagé en Israël par KATAN et al., dès 1976, et repris dans d'autres régions de même latitude géographique, (30-35 $\left.{ }^{\circ} \mathrm{L}\right)$ en Californie (JOHNSON et al.) en 1981, en Crète (TJAMOS \& FARIDIS) en 1980 et (MALATHRAKIS et al.) en 1983.

A ces latitudes, les températures relevées sous paillage en plein champ dépassent $50^{\circ} \mathrm{C}$ dans la zone superficielle et sont supérieures de 8 à $12^{\circ} \mathrm{C}$ à celles enregistrées en sol nu.

Nous avons recherché si ce procédé présentait de l'intérêt pour le littoral méditerranéen français malgré sa latitude plus élevée $\left(44^{\circ} \mathrm{L}\right)$ et son climat estival plus doux. A la différence des expérimentateurs précédents qui, dans un climat favorable, utilisent des films ordinaires, nous avons fait appel à des films plus performants et, comme GARIBALDI (1983) en Italie du Nord et GoISQUE et al. (1984), en France, nous les avons essayés sous serre, où les températures estivales 
sont particulièrement élevées et les problèmes de contamination des sols plus fréquents.

Les travaux exploratoires des étés 1979, 1980, 1981 ont permis de fixer la taille des parcelles et les types de film les plus performants dans leurs effets thermiques. L'expérience de 1982 a donné lieu à des analyses biologiques nombreuses et fréquentes pour quantifier l'action sur plusieurs éléments de la biocénose tellurique et analyser les processus de recolonisation.

\section{MATÉRIELS ET MÉTHODES}

\section{A. Caractéristiques des parcelles expérimentales}

Les essais sont implantés dans la zone littorale varoise, sur un sol sableux homogène sur toute la profondeur travaillée de 0 à $40 \mathrm{~cm}$, de richesse chimique moyenne, sans carence ou excès notables. La composition physique de ce sol est : 1 à $2 \mathrm{p} .100$ de matières organiques, 8 p. 100 d'argile, 12 p. 100 de limons, 79 p. 100 de sables.

La densité apparente du sol sec travaillé varie de 1,0 en surface à 1,35 environ à $25 \mathrm{~cm}$ de profondeur. Le coefficient calculé de chaleur spécifique du sol ressuyé est,

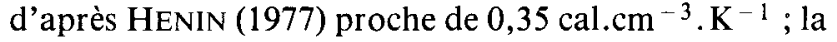
vitesse de propagation de l'onde thermique avoisine 2 à $3 \mathrm{~cm} \cdot \mathrm{h}^{-1}$.

La dimension des parcelles élémentaires, à l'air libre comme en serre, est passée de $3 \times 3 \mathrm{~m}$ en 1979 et 1980 , à $5 \times 5 \mathrm{~m}$ en 1981 , puis à $10 \times 10 \mathrm{~m}$ en 1982 , afin de limiter les déperditions latérales de chaleur et d'humidité.

Le sol désherbé et émietté par fraisage est arrosé à saturation avant la pose du film tendu et enterré en bordure (paillage). Cette opération est réalisée fin juin ou début juillet. Le paillage reste en place pendant 2 mois.

Les parcelles témoins non paillées sont désherbées périodiquement.

\section{B. Toiles plastiques éprouvées}

P.E. : Polyéthylène clair, d'épaisseur $150 \mu \mathrm{m}$.

E.V.A. : Copolymère d'éthylvinylacétate d'épaisseur $150 \mu \mathrm{m}$.

"Bulle »: Polyéthylène alvéolé, double paroi, utilisé pour l'isolation thermique des serres, le poids de matière au $\mathrm{m}^{2}$ est voisin de celui du film P.E., l'épaisseur de la couche d'air emprisonnée est de $5 \mathrm{~mm}$.

\section{Contrôle des résultats, prélèvements et analyses}

Les prélèvements de sol sont faits au centre des parcelles paillées ou témoins, à 4 profondeurs (0-10, 10-20, 20-30, 30-40 cm), avant paillage, puis $15 \mathrm{j}, 1$ et 2 mois après le début du traitement et 3 et 10 mois après la fin du traitement.

Les analyses nématologiques portent sur des échantillons de $1 \mathrm{~kg}$ de terre fine non desséchée ; elles sont réalisées par la méthode de flottation-centrifugation.

Pour les analyses microbiologiques, la méthode retenue est celle des dilutions à partir de $10 \mathrm{~g}$ de sol. Pour chaque prélèvement, $0,1 \mathrm{ml}$ des différentes dilutions est étalé sur un milieu nutritif solide en boîtes de Petri. Le milieu utilisé à 2 p. 100 de malt et 2 p. 100 de gélose per- met à la majorité des germes de se développer. Pour chaque dilution, 3 boîtes sont ensemencées et le dénombrement des micro-organismes est fait à partir du $4^{\mathrm{e}} \mathrm{j}$ d'incubation à $25^{\circ} \mathrm{C}$. La reconnaissance des espèces est faite soit directement sur l'ensemble des dilutions, soit après repiquage sur un autre milieu nutritif : (Avoine, Richard ou Sabouraud) afin d'obtenir les fructifications nécessaires à l'identification.

\section{OBSERVATIONS ET RÉSULTATS}

\section{A. Essais préliminaires 1979 et 1981}

Ces essais ont permis de préciser les impératifs à respecter pour accroître l'efficacité de cette méthode de désinfection. Ils peuvent se résumer ainsi :

- utilisation d'un plastique plus épais et plus performant du point de vue des échanges thermiques que le polyéthylène qui suffit dans les pays plus chauds : EVA, P.E. alvéolé « Bulle » ;

- nécessité d'obtenir des températures élevées aussitôt après le paillage pour éviter le développement des adventices ;

- choix de parcelles expérimentales de grande surface $(10 \times 10 \mathrm{~m})$ pour réduire les effets de bordure, notamment les pertes de chaleur ;

- difficultés d'emploi du procédé en serre fermée par suite des dommages occasionnés aux installations par l'action répétée des températures élevées.

\section{B. Essais 1982}

En plein air, le paillage «P.E. Bulle » est maintenu en place du 24 juin au 24 août après une jachère et, en serre, du 7 juillet au 5 septembre après une culture de gerbera.

Les données climatiques de l'été 1982 sont résumées dans le tableau 1. L'été 1982 a été particulièrement sec mais les températures de juillet à septembre sont proches des moyennes saisonnières.

\section{Effet sur les températures}

Les relevés réalisés au cours de l'essai (tabl. 2) montrent que, sous paillage, les moyennes, les maximums et les écarts thermiques sont, en plein air, supérieurs à ceux relevés en serre aérée et blanchie.

Les températures ont été mesurées en plein air après 2 mois de paillage jusqu'à $1,10 \mathrm{~m}$ de profondeur (fig. 1). Le profil de températures, fictif au-dessous de $1,10 \mathrm{~m}$, laisse supposer que l'effet du paillage atteint environ $3,50 \mathrm{~m}$ de profondeur.

\section{Effet sur l'humidité des sols}

L'évolution de l'humidité des sols dans les 4 traitements est indiquée dans le tableau 3 : l'humidité est restée très élevée même dans la couche superficielle des sols paillés.

\section{Effet sur la flore adventice}

Une réduction sensible du nombre de graines germantes dans le sol superficiel prélevé ultérieurement a 
TABLEAU 1

Principales données météorologiques - Fréjus été 1982 / année moyenne.

Summarized meteorological data - Fréjus summer 1982 / average data.

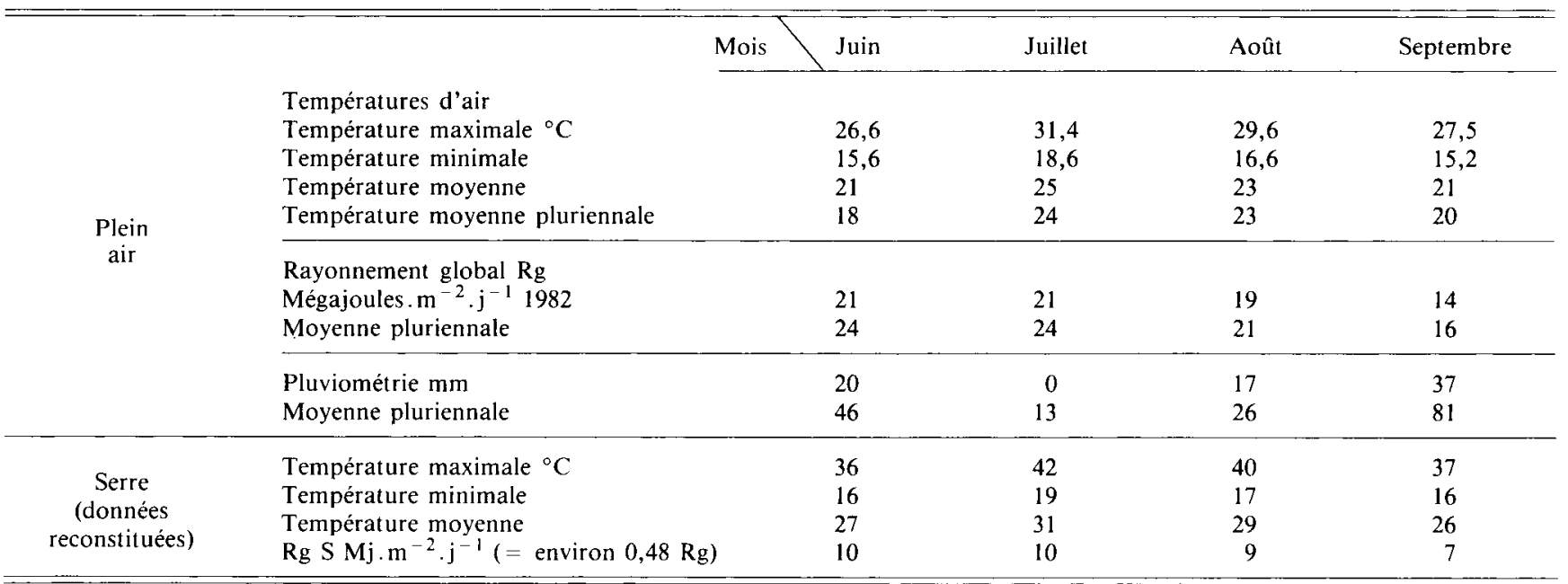

TABLEAU 2

Effets du paillage « Bulle » sur les températures du sol en plein air et en serre aérée blanchie Période du 15 juillet au 15 août 1982.

Effects of « Air-Bubble » mulch on soil temperatures outdoors and under glass (ventilated and whitewashed) Period of July 15 to August 15, 1982.

\begin{tabular}{|c|c|c|c|c|}
\hline & & Sol nu & Paille «Bulle» & Ecart \\
\hline \multicolumn{5}{|c|}{ Essai en plein air } \\
\hline Températures maximales observées à & $\begin{array}{l}-10 \mathrm{~cm} \\
-20 \mathrm{~cm}\end{array}$ & $38^{\circ} \mathrm{C}$ & $\begin{array}{l}47 \text { à } 49{ }^{\circ} \mathrm{C} \\
41 \text { à } 42{ }^{\circ} \mathrm{C}\end{array}$ & $+10^{\circ} \mathrm{C}$ \\
\hline Températures moyennes & $\begin{array}{l}-10 \mathrm{~cm} \\
-20 \mathrm{~cm}\end{array}$ & $29,8{ }^{\circ} \mathrm{C}$ & $\begin{array}{l}39,8{ }^{\circ} \mathrm{C} \\
38,3{ }^{\circ} \mathrm{C}\end{array}$ & $+10^{\circ} \mathrm{C}$ \\
\hline \multicolumn{5}{|l|}{ Essai en serre } \\
\hline Températures maximales observées à & $\begin{array}{l}-10 \mathrm{~cm} \\
-20 \mathrm{~cm}\end{array}$ & $35^{\circ} \mathrm{C}$ & $\begin{array}{c}44 \text { à } 45^{\circ} \mathrm{C} \\
39^{\circ} \mathrm{C}\end{array}$ & $+9,5^{\circ} \mathrm{C}$ \\
\hline Températures moyennes & $\begin{array}{l}-10 \mathrm{~cm} \\
-20 \mathrm{~cm}\end{array}$ & $29,7^{\circ} \mathrm{C}$ & $\begin{array}{l}38,8^{\circ} \mathrm{C} \\
35,7^{\circ} \mathrm{C}\end{array}$ & $+9,1{ }^{\circ} \mathrm{C}$ \\
\hline
\end{tabular}

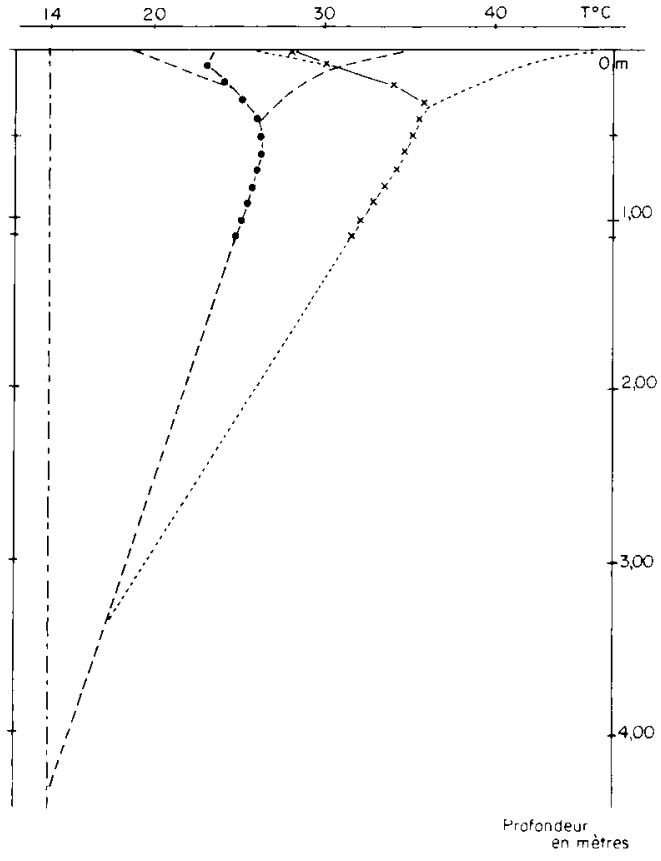

été observée : pour $100 \mathrm{~g}$ de sol sec, en plein air, on a obtenu :

66 plantules de Portulaca oleracea L. en sol nu, 27 en sol paillé,

8 plantules diverses en sol nu, aucune en sol paillé.

Figure 1

Profils thermiques en plein air, en sol nu et sol paillé (bulle).

Températures relevées le 24.08 .82 à 09 h T.L.

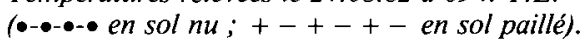

Prolongation théorique des profils au-dessous de 1,10 m de profondeur (---en sol nu; ....... en sol paillé) et variations journalières dans la zone superficielle (mêmes symboles).

-.- Température moyenne intersaisonnière théorique $=14^{\circ} \mathrm{C}$.

Temperature gradients of bare and " bubble "-mulched soil outdoors.

Temperatures measured on 24 Aug. 82 at 400.

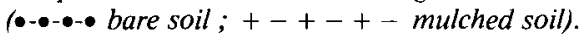

Theoretical gradients below $1.10 \mathrm{~m}$.

(---bare soil ; ...... mulched soil) and daily variations in the upper zone (same symbols).

- annual mean temperature $=14{ }^{\circ} \mathrm{C}$. 
TABLEAU 3

Effets du paillage “ Bulle " sur l'humidité des sols en plein air et en serre - été 1982 Humidité exprimée en pourcentage de l'humidité équivalente.

Effects of « Bubble " mulch on soil moisture outdoors and under glass - summer 1982 Moisture given as \% of equivalent humidity.

\begin{tabular}{|c|c|c|c|c|c|c|c|c|c|}
\hline Traitement & $\begin{array}{l}\text { Profondeurs } \\
\mathrm{cm}\end{array}$ & initial & $15^{\mathrm{e}} \mathrm{j}$ & $\begin{array}{l}\text { En plein air } \\
30^{\mathrm{e}} \mathrm{j}\end{array}$ & $60^{e} j$ & $146^{\mathrm{e}} \mathrm{j}$ & initial & $\begin{array}{c}\text { En serre } \\
30^{\mathrm{e}} \mathrm{j}\end{array}$ & $60^{c} j$ \\
\hline \multirow[t]{4}{*}{ Témoin } & $0-10$ & $71 \%$ & 48 & 25 & 22 & $>110$ & 111 & 36 & 41 \\
\hline & $10-20$ & 88 & 74 & 44 & 71 & $"$ & 120 & 66 & 57 \\
\hline & $20-30$ & 92 & & 55 & 43 & $"$ & 93 & 77 & 67 \\
\hline & $30-40$ & 94 & & 76 & 54 & $"$ & 75 & 83 & 73 \\
\hline \multirow{3}{*}{ Paillé } & $10-20$ & 88 & 85 & 92 & 82 & " & 120 & 98 & 95 \\
\hline & $20-30$ & 92 & & 88 & 81 & $"$ & 93 & 101 & 98 \\
\hline & $30-40$ & 94 & & 96 & 82 & $"$ & 75 & 99 & 96 \\
\hline
\end{tabular}

Le peuplement végétal spontané de ces parcelles, en mai 1983, est également différencié.

\section{Effet sur la nématofaune}

\section{a) Essai de plein air}

\section{- Après 15 jours de solarisation}

Dans l'ensemble des parcelles de plein air, 47 espèces de nématodes sont identifiées. Le tableau 4 en donne la répartition par groupes trophiques avant et $15 \mathrm{j}$ après la mise en place du paillage.

\section{TABLEAU 4}

Répartition des nématodes selon leur groupe trophique avant le début des essais et 15 jours plus tard en sol de plein air nu ou paillé (Film «Bulle »).

Trophic groups of nematodes found initially and after 15 days in mulched and unmulched soil outdoors.

\begin{tabular}{llccc}
\hline \hline Groupes trophiques & $\begin{array}{c}\text { Avant } \\
\text { traitement }\end{array}$ & $\begin{array}{c}\text { Témoin } \\
\text { nu }\end{array}$ & $\begin{array}{c}\text { Parcelle } \\
\text { paillée }\end{array}$ \\
\hline Phytophages & 24 espèces & & 23 & 7 \\
Bactériophages & 10 & 9 & 6 \\
Créophages & 4 & & 4 & 1 \\
Mycophages & 1 & & 1 & 1 \\
Omnivores & 8 & & 6 & 6 \\
\cline { 2 - 2 }$\quad$ Total & 47 & & 43 & \\
\hline
\end{tabular}

La diversité spécifique est donc rapidement et nettement réduite par la solarisation : les phytophages et les créophages semblent les plus sensibles. Cette sélectivité est surtout due à la localisation superficielle de ces 2 groupes. En fait, la réduction drastique des effectifs atténue beaucoup les conséquences de cette sélectivité. Si l'on considère en effet les populations retrouvées dans les 2 horizons échantillonnés après $15 \mathrm{j}$ (fig. 2), on observe un accroissement de presque tous les groupes trophiques dans les parcelles témoins par rapport à l'état initial et une réduction dans des proportions considérables des espèces représentées et des effectifs de tous les nématodes sous paillage, bien que cette action ait été moins marquée au-dessous de $10 \mathrm{~cm}$; un seul phytophage a été décelé dans l'horizon -10 à $-20 \mathrm{~cm}$ appartenant aux Tylenchus, genre dont la nuisibilité pour les végétaux est incertaine.

\section{- 1 mois plus tard}

Dans les parcelles témoins, la réduction des populations est d'environ 50 p. 100 dans la couche superficielle, ce qui s'explique par l'absence prolongée de couvert végétal et la dessiccation du sol.

Sous paillage, la couche superficielle déjà très affectée après $15 \mathrm{j}$ ne paraît pas sensiblement différente. Toutefois, les phytophages et surtout les bactériophages sont nettement moins nombreux entre -10 et $-20 \mathrm{~cm}$ que lors du contrôle précédent.

Les couches inférieures sont plus peuplées, en raison surtout de Paratylenchus curvitatus Van der Linde s.l., espèce susceptible de coloniser des horizons très profonds s'ils sont exploités par des racines.

\section{-2 mois plus tard}

Dans les parcelles témoins, la situation nématologique n'est pas sensiblement modifiée, en dehors d'une recrudescence des phytophages, malgré l'absence entretenue de végétation.

Les principaux phytophages responsables de ce repeuplement appartiennent aux espèces :

Tylenchus sp., Boleodorus thylactus Thorne, Neopsilenchus sp., Merlinius microdorus (Geraert) Siddigi, Merlinius brevidens (Allen) Siddigi, Rotylenchus laurentinus Scognamiglio \& Talame, Paratylenchus curvitatus s.l., Trichodorus primitivus (de Man) Micoletzky.

Dans les parcelles paillées, le dépeuplement est quasi total sous plastique, il est moins marqué dans les couches profondes, notamment du fait des bactériophages (Acrobeles ciliatus Linstow et Acrobeloides (Cobb Thorne) et des mycophages (Aphelenchus avenae Bastian).

Une série d'analyses faites un an après le début de l'essai, c'est-à-dire 10 mois après l'enlèvement des plastiques, révèle (tabl. 5) une persistance remarquable des effets du paillage sur tous les groupes trophiques et aux 4 horizons étudiés, bien qu'une recolonisation très importante ait été enregistrée au cours des 10 mois qui ont suivi le paillage. Cette persistance se traduit par une réduction de 95 p. 100 des populations initiales. 

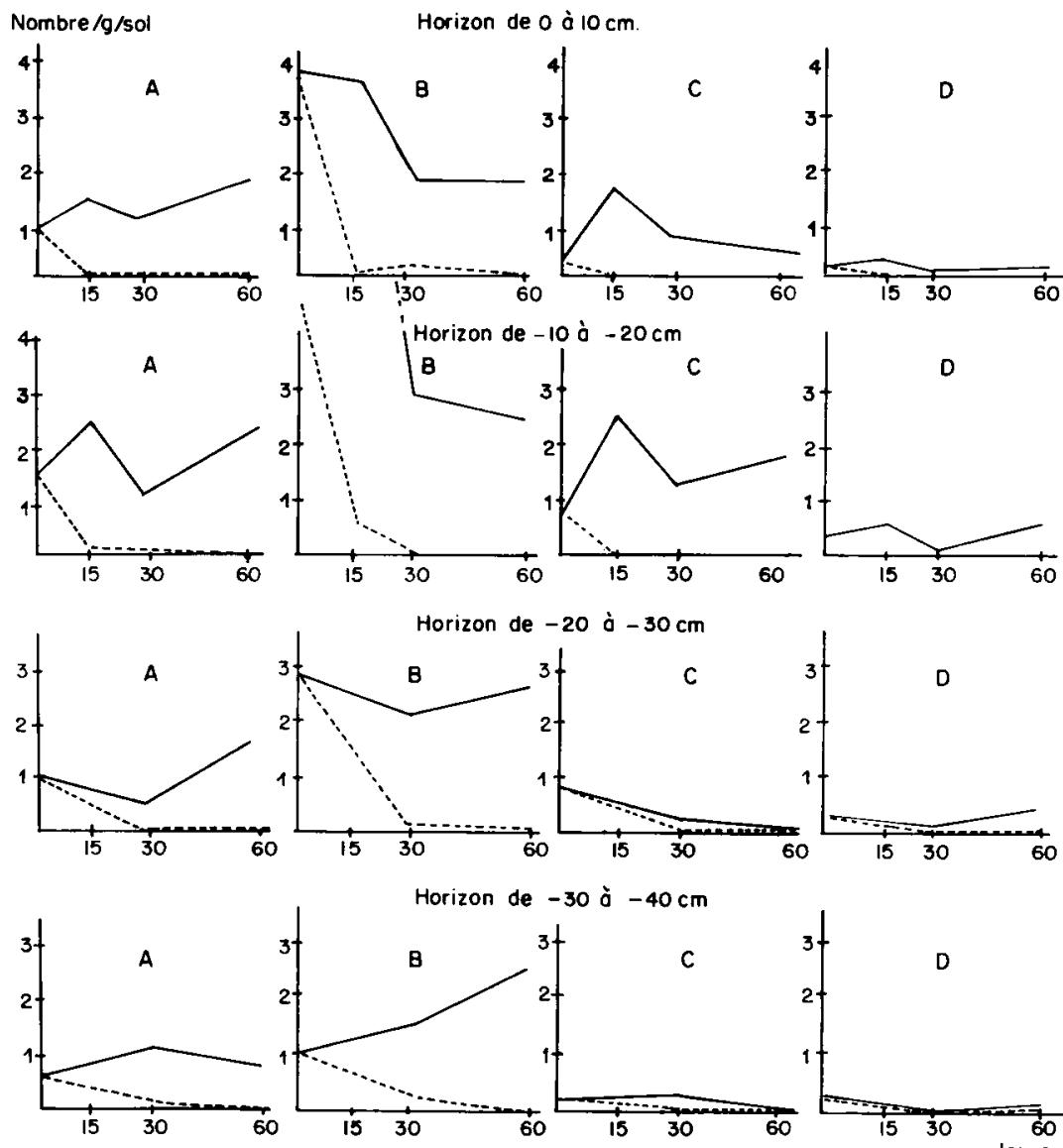

A : Phytophages

B : Bactériophages

C: Mycophages

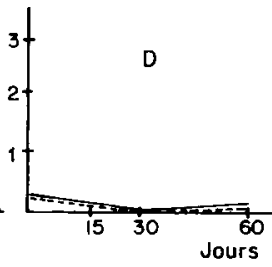

Figure 2

Plastic mulching effects on the four trophic groups of nematodes in an outdoor soil (given in number of nematodes per $g$ of soil) (Fréjus, France, summer 1982).

duce paillage sur les effectifs de 4 groupes trophiques d'un sol de plein air exprimée en nombre de nématodes par $g$ de sol (Fréjus,

Paillage bulle (Plastic bubble mulching)

Témoin non paillé (No mulching check)

TABLEAU 5

Nématodes retrouvés dans les 4 horizons d'un sol de plein air 10 mois après l'enlèvement du paillage

A. Effectifs dénombrés (individus par $100 \mathrm{~g}$ de sol)

B. Pourcentages par rapport aux effectifs à l'enlèvement du paillage $(60 \mathrm{j})$

C. Pourcentages par rapport aux effectifs initiaux (mise en place du paillage $j 0$ ).

Numbers of nematodes in the four layers of a soil outdoors 10 months after removal of mulch

A. Number of individuals per $100 \mathrm{~g}$ of soil

B. \% of the population on day 60 (mulch removal)

C. \% of initial population.

\begin{tabular}{|c|c|c|c|c|c|c|}
\hline \multicolumn{2}{|c|}{ Groupes trophiques } & \multirow[t]{2}{*}{ Phytophages } & \multirow[t]{2}{*}{ Bactériophages } & \multirow[t]{2}{*}{ Mycophages } & \multirow[t]{2}{*}{ Omnivores } & \multirow[t]{2}{*}{ Total } \\
\hline \multicolumn{2}{|l|}{ Horizon } & & & & & \\
\hline \multirow{3}{*}{$0-10 \mathrm{~cm}$} & A & 11 & 25 & + & + & 36 \\
\hline & B & $>1100$ & 800 & $<33$ & $(100)$ & 600 \\
\hline & $\mathrm{C}$ & 15 & 7 & $<4$ & $<8$ & 7 \\
\hline \multirow{4}{*}{$10-20 \mathrm{~cm}$} & A & 3 & 43 & 3 & 3 & 52 \\
\hline & B & $\infty$ & 1400 & 100 & $\infty$ & 800 \\
\hline & $\mathrm{C}$ & 2 & 9 & 4 & 7 & 7 \\
\hline & $A$ & + & 13 & 3 & 3 & 16 \\
\hline \multirow{3}{*}{$20-30 \mathrm{~cm}$} & B & $\infty$ & 70 & 33 & $>300$ & 57 \\
\hline & $\mathrm{C}$ & 1 & 4 & 4 & 11 & 3 \\
\hline & A & 3 & 33 & 0 & 3 & 39 \\
\hline \multirow[t]{2}{*}{$30-40 \mathrm{~cm}$} & B & $\infty$ & 200 & 0 & 100 & 130 \\
\hline & $\mathrm{C}$ & 5 & 33 & 0 & 13 & 19 \\
\hline
\end{tabular}

$+=$ moins d'un individu par $100 \mathrm{~g}$ de sol.

$+=$ less than 1 individual per $100 \mathrm{~g}$ of soil. 
TABLEAU 6

Action d'un paillage plastique "Bulle » sur la nématofaune d'un sol de serre - été 1982 Effectifs exprimés en nombre d'individus par $100 \mathrm{~g}$ de sol.

Effects of "Bubble " mulch on the nematofauna of a glasshouse soil - summer 1982 Numbers of individuals per $100 \mathrm{~g}$ of soil.

\begin{tabular}{|c|c|c|c|c|c|c|c|c|c|c|c|c|c|c|c|}
\hline \multirow{2}{*}{$\begin{array}{l}\text { Groupes } \\
\text { trophiques }\end{array}$} & \multirow{2}{*}{\multicolumn{2}{|c|}{$\begin{array}{c}\text { Nombre } \\
\text { d'espèces } \\
\text { identifiées }\end{array}$}} & \multirow{2}{*}{$\begin{array}{c}\text { Profondeurs } \\
\text { Durée du } \\
\text { traitement }\end{array}$} & \multicolumn{3}{|c|}{0 à $10 \mathrm{~cm}$} & \multicolumn{3}{|c|}{10 à $20 \mathrm{~cm}$} & \multicolumn{3}{|c|}{20 à $30 \mathrm{~cm}$} & \multicolumn{3}{|c|}{30 à $40 \mathrm{~cm}$} \\
\hline & & & & $\begin{array}{l}\text { état } \\
\text { initial }\end{array}$ & $30 \mathrm{j}$ & $60 \mathrm{j}$ & $\begin{array}{l}\text { état } \\
\text { initial }\end{array}$ & $30 \mathrm{j}$ & $60 \mathrm{j}$ & $\begin{array}{l}\text { état } \\
\text { initial }\end{array}$ & $30 \mathrm{j}$ & $60 \mathrm{j}$ & $\begin{array}{l}\text { état } \\
\text { initial }\end{array}$ & $30 j$ & $60 \mathrm{j}$ \\
\hline \multirow{2}{*}{ Bactériophages } & \multirow{2}{*}{10} & 10 & nu & \multirow{2}{*}{303} & 55 & 208 & \multirow{2}{*}{221} & 38 & 153 & \multirow{2}{*}{147} & 53 & 98 & \multirow{2}{*}{118} & 83 & 68 \\
\hline & & 5 & paillé «Bulle " & & 40 & 15 & & 58 & 53 & & 40 & 75 & & 85 & 63 \\
\hline \multirow[b]{2}{*}{ Phytophages } & \multirow[b]{2}{*}{8} & 6 & nu & \multirow[b]{2}{*}{0} & 0 & 0 & \multirow[b]{2}{*}{0} & 0 & 0 & \multirow[b]{2}{*}{+} & 0 & + & \multirow[b]{2}{*}{9} & + & 0 \\
\hline & & 3 & paillé «Bulle " & & 0 & 0 & & 0 & 0 & & + & 0 & & 8 & + \\
\hline \multirow[b]{2}{*}{ Mycophages } & \multirow[b]{2}{*}{2} & 2 & nu & \multirow[b]{2}{*}{15} & + & 8 & \multirow[b]{2}{*}{+} & + & 8 & \multirow[b]{2}{*}{0} & 3 & 10 & \multirow[b]{2}{*}{9} & 13 & 10 \\
\hline & & 1 & paillé «Bulle » & & 0 & 3 & & 5 & 10 & & 5 & 8 & & 13 & 8 \\
\hline \multirow[b]{2}{*}{ Omnivores } & \multirow[b]{2}{*}{2} & 2 & nu & \multirow[b]{2}{*}{+} & 0 & 3 & \multirow[b]{2}{*}{+} & 0 & + & \multirow[b]{2}{*}{3} & 3 & + & \multirow[b]{2}{*}{3} & 3 & + \\
\hline & & 2 & paillé "Bulle " & & 3 & + & & 5 & 0 & & + & + & & + & 3 \\
\hline \multirow{2}{*}{ Population tolale } & \multirow[b]{2}{*}{22} & 20 & nu & \multirow{2}{*}{318} & 55 & 219 & \multirow[b]{2}{*}{221} & 38 & 161 & & 59 & 108 & & 99 & 78 \\
\hline & & 11 & paillé "Bulle " & & 43 & 18 & & 68 & 63 & 150 & 45 & 83 & 139 & 106 & 74 \\
\hline
\end{tabular}

(+) moins de 1 individu par 100 grammes de sol.

$(+)$ less than 1 individual per $100 \mathrm{~g}$ of soil.

\section{b) Essai sous serre}

Le résultat des analyses montre (tabl. 6) que la nématofaune des sols de serres est extrêmement pauvre, tant en genres qu'en espèces. Les différences les plus accusées entre les 2 traitements apparaissent dans la couche superficielle et ne concernent que les bactériophages. Elles ne sont plus significatives au-dessous de $20 \mathrm{~cm}$ et ne se manifestent nettement en surface que dans les contrôles effectués 2 mois après le début du paillage.

De l'ensemble des résultats obtenus, tant en serre qu'en plein air, il est intéressant de noter au plan nématologique :

- Que dans la zone superficielle, où les variations journalières de température permettent de dépasser $45^{\circ} \mathrm{C}$ pendant plusieurs heures, l'analyse révèle toujours des nématodes, alors que $43,5^{\circ} \mathrm{C}$ est considéré comme létal pour la plupart des stades mobiles.

- Que dans la zone de - 20 à $-40 \mathrm{~cm}$, où la température moyenne s'établit sous paillage aux environs de $35^{\circ} \mathrm{C}$, soit à $10{ }^{\circ} \mathrm{C}$ au-dessus des conditions naturelles, un effet net est obtenu après 1 , ou mieux 2 mois d'application. Une telle élévation modérée mais de longue durée de la température pourrait donc agir également dans les couches plus profondes, que nous n'avons pas jugé utile, peut-être à tort, d'analyser au cours de ces essais.

Si le paillage plastique estival permet donc d'éliminer la quasi-totalité des nématodes situés dans les couches superficielles et jusqu'à $40 \mathrm{~cm}$ de protondeur, ce procédé s'avère assez peu sélectif.

Toutefois, parmi les 5 groupes trophiques composant la nématofaune, les bactériophages, suivis des mycophages, paraissent les moins affectés par les températures élevées. A l'opposé, les phytophages, en dehors de ceux localisés dans les couches profondes, sont plus sensibles, en raison peut-être aussi de l'absence de source alimentaire.

Parmi les 74 espèces identifiées, celles qui résistent en plus grand nombre à ce traitement appartiennent aux genres bactériophages $A$ crobeles ( $A$. ciliatus) et Acrobeloides, et mycophages (Aphelenchus avenae). Parmi les phytophages, 4 genres sont retrouvés sous les paillages en fin d'essai : Paratylenchus ( $P$. curvitatus et $P$. baldacci Raski), Merlinius ( $M$. microdorus et $M$. brevidens), Rotylenchus ( $R$. laurentinus), Trichodorus ( $T$. viruliferus Hooper).

Les examens réalisés 10 mois après l'enlèvement du paillage montrent que cet effet est durable (tabl. 7).

\section{TABLEAU 7}

Répartition des nématodes selon leur groupe trophique avant le début des essais et 10 mois plus tard en sol de serre nu ou paillé (Film «Bulle »).

Trophic groups of nematodes found initially and after 10 months in mulched and unmulched glasshouse soil.

\begin{tabular}{lccc}
\hline Groupes trophiques & $\begin{array}{c}\text { Avant } \\
\text { traitement }\end{array}$ & $\begin{array}{c}\text { Témoin } \\
\text { nu }\end{array}$ & $\begin{array}{c}\text { Parcelle } \\
\text { paillée }\end{array}$ \\
\hline Phytophages & 8 espèces & 6 & 3 \\
Bactériophages & 10 & 10 & 5 \\
Créophages & 0 & 0 & 0 \\
Mycophages & 2 & 2 & 1 \\
Omnivores & 2 & 2 & 2 \\
\cline { 3 - 4 }$\quad$ Total & 22 & 20 & 11 \\
\hline
\end{tabular}

\section{Effets sur la microflore}

Les populations bactériennes, de loin les plus importantes, sont à l'origine identiques en serre et à 
l'extérieur, jusqu'à $20 \mathrm{~cm}$ de profondeur. Au-delà, elles sont nettement plus pauvres sous serre. Il en est de même pour la flore mycologique et les actinomycètes au-dessous de $10 \mathrm{~cm}$.

En ce qui concerne les bactéries (fig. 3-A), on observe en plein air une diminution marquée des populations dès le $15^{\mathrm{e}} \mathrm{j}$. Cet effet temporairement inversé au $30^{\mathrm{e}} \mathrm{j}$ jusqu'à $20 \mathrm{~cm}$ se poursuit jusqu'au $60^{\mathrm{e}} \mathrm{j}$, puis tend à s'estomper. Le paillage en accroît généralement l'intensité.

En serre, l'effet est inverse mais, après une augmentation des effectifs durant le $1^{\text {er }}$ mois, la tendance s'infléchit. Le paillage amplifie ici encore le phénomène.

Sur la microflore fongique, incluant les actinomycètes (fig. 3-B) l'effet de désinfection solaire est le plus marqué. On observe la disparition de certaines populations pendant des temps variables, notamment sous paillage en plein air.

L'effet de désinfection est moins marqué sous serre qu'en plein air.

Lorsqu'on considère l'ensemble des courbes d'évolution des composants de la microflore, le fait le plus marquant est l'incidence certaine de la désinfection solaire sur les populations des horizons $-10 \mathrm{~cm}$ à $-40 \mathrm{~cm}$.

Pour ce qui est du nombre de genres microbiens isolés dans les différents traitements, on ne constate pas de différence, en serre, entre les sols nu et paillé ; par contre, en plein air, le paillage diminue fortement et durablement la diversité de la flore. Seuls subsistent les Penicillium, Aspergillus et Fusarium, dont certaines espèces sont connues pour résister à la chaleur (tabl. 8).

Enfin, les examens effectués $146 \mathrm{j}$ après le début de l'essai, soit 3 mois environ après l'enlèvement du film, montrent que la recolonisation par les cryptogames conserve les différences relevées pendant le paillage.

\section{CONCLUSION}

Le paillage estival des sols procure dans le Sud de la France des effets biologiques tout à fait comparables à ceux mentionnés dans les pays plus chauds. Les conditions favorables à sa pleine efficacité (insolation régulière et continue surtout durant la $1^{\text {re }}$ semaine
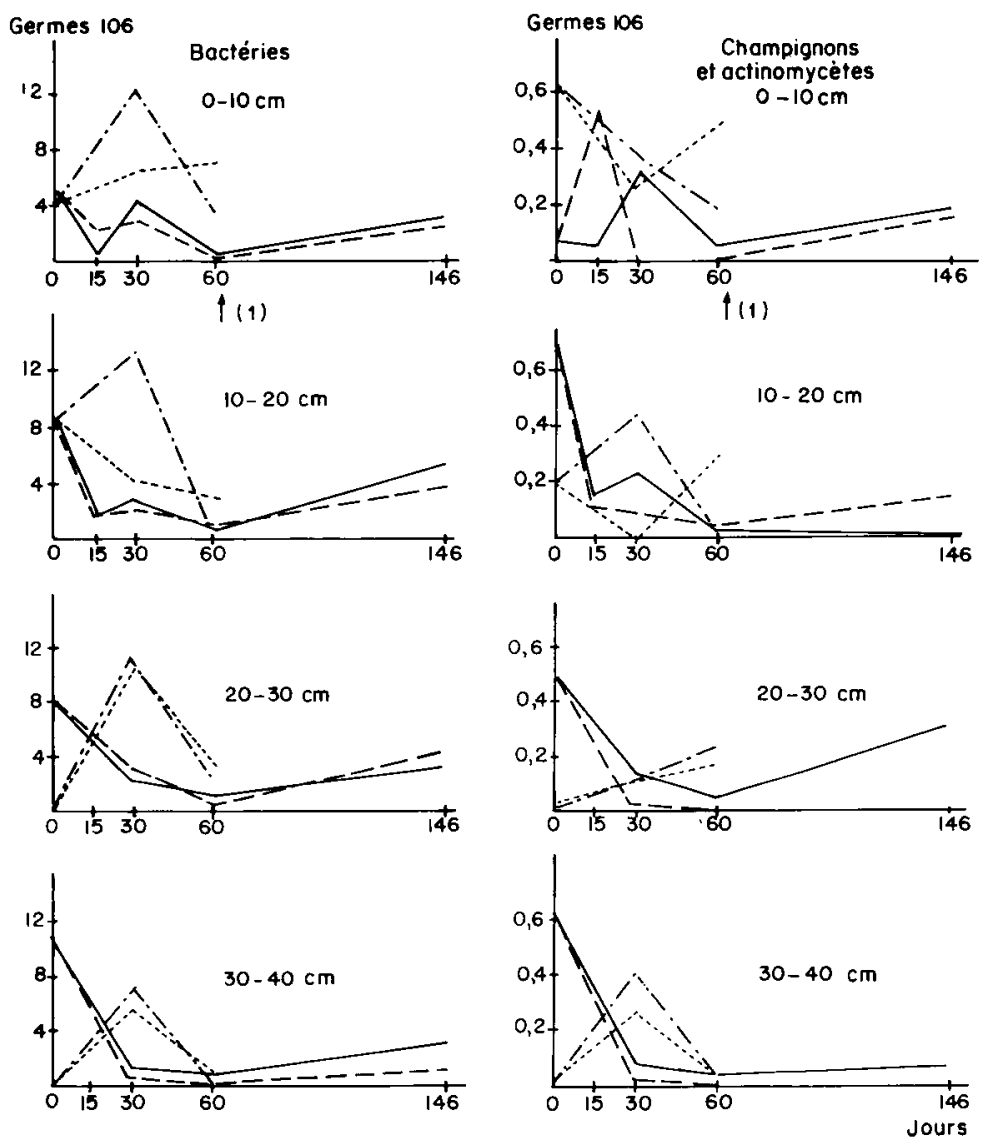

A: population boctérienne

(1) Enlèvement du paillage le $60^{\mathrm{e}}$ jour Mulch removed on $60^{\text {th }}$ day

mulch. Fréjus, summer 1982. Number of colony-forming units per $g$ of dry soil.

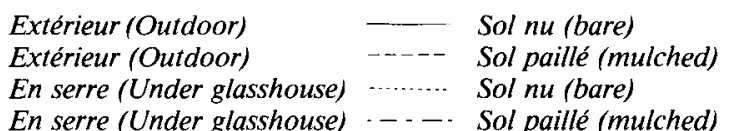


TABLEAU 8

Genres microbiens isolés des sols à différents traitements - été 1982.

Microbial genera isolated from soils with different treatments - summer 1982.

\begin{tabular}{|c|c|c|c|c|c|c|c|c|c|c|c|c|c|c|c|c|c|c|c|c|c|c|c|c|c|c|c|c|c|c|c|}
\hline \multirow{3}{*}{$\begin{array}{l}\text { Localication } \\
\text { Profondeur de prélèvement } \\
\text { Date de prélèvement }\end{array}$} & & \multicolumn{18}{|c|}{ Plein air } & \multicolumn{12}{|c|}{ Serre } \\
\hline & & \multicolumn{5}{|c|}{$0-10 \mathrm{~cm}$} & \multicolumn{5}{|c|}{$10-20 \mathrm{~cm}$} & \multicolumn{4}{|c|}{$20-30 \mathrm{~cm}$} & \multicolumn{4}{|c|}{$30-40 \mathrm{~cm}$} & \multicolumn{3}{|c|}{$0-10 \mathrm{~cm}$} & \multicolumn{3}{|c|}{$10-20 \mathrm{~cm}$} & \multicolumn{3}{|c|}{$20-30 \mathrm{~cm}$} & \multicolumn{3}{|c|}{$30-40 \mathrm{~cm}$} \\
\hline & & 0 & 15 & 30 & 60 & 146 & 0 & 15 & 30 & 60 & 146 & 0 & 30 & 60 & 146 & 0 & 30 & 60 & 146 & 0 & 30 & 60 & 0 & 30 & 60 & 0 & 30 & 60 & 0 & 30 & 60 \\
\hline Bactéries & $\begin{array}{c}\text { nu } \\
\text { bulle }\end{array}$ & + & + & $\begin{array}{l}+ \\
+\end{array}$ & $\begin{array}{l}+ \\
+\end{array}$ & $\begin{array}{l}+ \\
+\end{array}$ & + & $\begin{array}{l}+ \\
+\end{array}$ & $\begin{array}{l}+ \\
+\end{array}$ & $\begin{array}{l}+ \\
+\end{array}$ & $\begin{array}{l}+ \\
+\end{array}$ & + & $\begin{array}{l}+ \\
+\end{array}$ & $\begin{array}{l}+ \\
+\end{array}$ & $\begin{array}{l}+ \\
+\end{array}$ & + & $\begin{array}{l}+ \\
+\end{array}$ & $\begin{array}{l}+ \\
+\end{array}$ & $\begin{array}{l}+ \\
+\end{array}$ & + & $\begin{array}{l}+ \\
+\end{array}$ & $\begin{array}{l}+ \\
+\end{array}$ & + & + & $\begin{array}{l}+ \\
+\end{array}$ & + & $\begin{array}{l}+ \\
+\end{array}$ & $\begin{array}{l}+ \\
+\end{array}$ & + & $\begin{array}{l}+ \\
+\end{array}$ & $\begin{array}{l}+ \\
+\end{array}$ \\
\hline Actinomycètes & $\begin{array}{l}\mathbf{n} \\
\mathrm{b}\end{array}$ & + & - & $\begin{array}{l}+ \\
-\end{array}$ & $\begin{array}{l}- \\
-\end{array}$ & $\begin{array}{l}+ \\
-\end{array}$ & + & $\begin{array}{l}- \\
-\end{array}$ & $\begin{array}{l}+ \\
+\end{array}$ & $\begin{array}{l}- \\
-\end{array}$ & + & + & + & $\begin{array}{l}- \\
-\end{array}$ & + & + & + & $\begin{array}{l}- \\
+\end{array}$ & $\begin{array}{l}+ \\
-\end{array}$ & + & + & + & + & + & $\begin{array}{l}+ \\
+\end{array}$ & + & $\begin{array}{l}- \\
-\end{array}$ & $\begin{array}{l}+ \\
+\end{array}$ & - & $\begin{array}{l}+ \\
+\end{array}$ & $\begin{array}{l}+ \\
+\end{array}$ \\
\hline Alternaria sp. & $\begin{array}{l}n \\
b\end{array}$ & + & $\begin{array}{l}- \\
-\end{array}$ & $\begin{array}{l}+ \\
-\end{array}$ & $\begin{array}{l}- \\
-\end{array}$ & $\begin{array}{l}- \\
-\end{array}$ & - & $\begin{array}{l}- \\
-\end{array}$ & + & - & - & $\cdots$ & $\begin{array}{l}- \\
-\end{array}$ & $\begin{array}{l}+ \\
-\end{array}$ & $\begin{array}{l}- \\
-\end{array}$ & + & $\begin{array}{l}- \\
-\end{array}$ & $\begin{array}{l}- \\
-\end{array}$ & $\begin{array}{l}- \\
-\end{array}$ & - & $\begin{array}{l}- \\
-\end{array}$ & + & - & $\begin{array}{l}- \\
-\end{array}$ & - & - & $\begin{array}{l}- \\
-\end{array}$ & $\begin{array}{l}- \\
-\end{array}$ & - & - & + \\
\hline Aspergillus sp. & $\begin{array}{l}\mathrm{n} \\
\mathrm{b}\end{array}$ & + & $\overline{+}$ & + & $\begin{array}{l}+ \\
-\end{array}$ & $\begin{array}{l}+ \\
-\end{array}$ & + & $\begin{array}{l}+ \\
+\end{array}$ & $\begin{array}{l}+ \\
+\end{array}$ & $\begin{array}{l}+ \\
+\end{array}$ & + & + & $\begin{array}{l}+ \\
+\end{array}$ & + & + & + & $\begin{array}{l}+ \\
+\end{array}$ & + & + & - & $\begin{array}{l}+ \\
+\end{array}$ & + & - & $\begin{array}{l}+ \\
+\end{array}$ & + & - & + & $\begin{array}{l}+ \\
+\end{array}$ & - & $\begin{array}{l}+ \\
+\end{array}$ & + \\
\hline Cephalosporium sp. & $\begin{array}{l}\mathrm{n} \\
\mathrm{b}\end{array}$ & - & $\begin{array}{l}- \\
-\end{array}$ & $\begin{array}{l}- \\
-\end{array}$ & $\begin{array}{l}- \\
-\end{array}$ & $\begin{array}{l}- \\
-\end{array}$ & - & $\begin{array}{l}- \\
-\end{array}$ & $\begin{array}{l}+ \\
-\end{array}$ & $\begin{array}{l}- \\
-\end{array}$ & $\begin{array}{l}- \\
+\end{array}$ & + & - & $\begin{array}{l}- \\
-\end{array}$ & $\begin{array}{l}- \\
-\end{array}$ & + & $\begin{array}{l}- \\
-\end{array}$ & $\begin{array}{l}- \\
-\end{array}$ & - & - & + & $\begin{array}{l}- \\
-\end{array}$ & - & $\begin{array}{l}- \\
-\end{array}$ & - & - & + & $\begin{array}{l}- \\
-\end{array}$ & - & - & - \\
\hline Cladosporium sp. & $\begin{array}{l}\mathrm{n} \\
\mathbf{b}\end{array}$ & - & 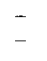 & $\begin{array}{l}+ \\
-\end{array}$ & $\begin{array}{l}- \\
-\end{array}$ & $\begin{array}{l}- \\
-\end{array}$ & + & $\begin{array}{l}+ \\
+\end{array}$ & + & - & - & + & - & - & $\begin{array}{l}- \\
-\end{array}$ & + & $\begin{array}{l}- \\
-\end{array}$ & $\begin{array}{l}- \\
-\end{array}$ & $\begin{array}{l}- \\
-\end{array}$ & - & - & $\begin{array}{l}- \\
-\end{array}$ & + & $\begin{array}{l}- \\
-\end{array}$ & - & - & + & $\begin{array}{l}- \\
-\end{array}$ & + & + & + \\
\hline Chalaropsis sp. & $\begin{array}{l}n \\
b\end{array}$ & + & $\begin{array}{l}- \\
-\end{array}$ & + & - & $\begin{array}{l}- \\
-\end{array}$ & - & $\begin{array}{l}- \\
-\end{array}$ & $\begin{array}{l}- \\
-\end{array}$ & - & - & - & $\begin{array}{l}- \\
-\end{array}$ & $\begin{array}{l}- \\
-\end{array}$ & - & - & - & - & $\begin{array}{l}- \\
-\end{array}$ & - & $\begin{array}{l}- \\
-\end{array}$ & $\begin{array}{l}- \\
-\end{array}$ & - & - & - & - & - & $\begin{array}{l}- \\
-\end{array}$ & - & - & - \\
\hline Didymostilbe $s p$. & $\begin{array}{l}n \\
b\end{array}$ & - & $\begin{array}{l}- \\
-\end{array}$ & $\begin{array}{l}- \\
-\end{array}$ & $\begin{array}{l}- \\
-\end{array}$ & $\begin{array}{l}- \\
-\end{array}$ & - & $\begin{array}{l}- \\
-\end{array}$ & $\begin{array}{l}- \\
-\end{array}$ & $\begin{array}{l}- \\
-\end{array}$ & - & - & $\begin{array}{l}- \\
-\end{array}$ & $\begin{array}{l}- \\
-\end{array}$ & $\begin{array}{l}- \\
-\end{array}$ & - & $\begin{array}{l}- \\
-\end{array}$ & + & $\begin{array}{l}- \\
-\end{array}$ & - & $\begin{array}{l}- \\
-\end{array}$ & $\begin{array}{l}- \\
-\end{array}$ & - & - & $\begin{array}{l}- \\
-\end{array}$ & - & $\begin{array}{l}- \\
-\end{array}$ & - & - & - & - \\
\hline Epicoccum sp. & $\begin{array}{l}n \\
b\end{array}$ & + & $\begin{array}{l}- \\
-\end{array}$ & $\begin{array}{l}- \\
-\end{array}$ & + & - & - & $\begin{array}{l}- \\
-\end{array}$ & $\begin{array}{l}- \\
-\end{array}$ & + & - & - & $\begin{array}{l}- \\
-\end{array}$ & $\begin{array}{l}+ \\
-\end{array}$ & $\begin{array}{l}- \\
-\end{array}$ & - & $\begin{array}{l}- \\
-\end{array}$ & $\begin{array}{l}- \\
-\end{array}$ & $\begin{array}{l}- \\
-\end{array}$ & - & $\begin{array}{l}- \\
-\end{array}$ & $\begin{array}{l}- \\
-\end{array}$ & - & - & - & - & $\begin{array}{l}- \\
-\end{array}$ & $\begin{array}{l}- \\
-\end{array}$ & + & $\begin{array}{l}- \\
-\end{array}$ & $\begin{array}{l}- \\
-\end{array}$ \\
\hline Fusarium sp. & $\begin{array}{l}n \\
b\end{array}$ & + & - & $\begin{array}{l}+ \\
-\end{array}$ & $\begin{array}{l}+ \\
+\end{array}$ & $\begin{array}{l}+ \\
-\end{array}$ & + & $\begin{array}{l}+ \\
+\end{array}$ & $\begin{array}{l}+ \\
-\end{array}$ & $\begin{array}{l}+ \\
-\end{array}$ & $\begin{array}{l}+ \\
-\end{array}$ & + & + & $\begin{array}{l}+ \\
-\end{array}$ & $\begin{array}{l}+ \\
+\end{array}$ & + & + & $\begin{array}{l}+ \\
+\end{array}$ & $\begin{array}{l}+ \\
+\end{array}$ & - & $\begin{array}{l}- \\
-\end{array}$ & + & - & $\overline{+}$ & $\begin{array}{l}+ \\
-\end{array}$ & - & $\overline{+}$ & $\begin{array}{l}+ \\
+\end{array}$ & - & $\begin{array}{l}- \\
-\end{array}$ & $\begin{array}{l}- \\
-\end{array}$ \\
\hline Geotrichum sp. & $\begin{array}{l}n \\
b\end{array}$ & - & $\begin{array}{l}- \\
-\end{array}$ & $\begin{array}{l}- \\
-\end{array}$ & $\begin{array}{l}- \\
-\end{array}$ & $\begin{array}{l}- \\
-\end{array}$ & + & $\begin{array}{l}- \\
-\end{array}$ & $\begin{array}{l}- \\
-\end{array}$ & - & $\begin{array}{l}- \\
-\end{array}$ & - & - & $\begin{array}{l}- \\
-\end{array}$ & $\begin{array}{l}- \\
-\end{array}$ & - & $\begin{array}{l}- \\
-\end{array}$ & $\begin{array}{l}- \\
-\end{array}$ & $\begin{array}{l}- \\
-\end{array}$ & - & - & - & - & $\begin{array}{l}- \\
-\end{array}$ & - & - & - & - & - & - & - \\
\hline Gliōcladium sp. & $\begin{array}{l}n \\
\text { b }\end{array}$ & - & $\begin{array}{l}- \\
-\end{array}$ & $\begin{array}{l}- \\
-\end{array}$ & $\begin{array}{l}- \\
-\end{array}$ & $\begin{array}{l}- \\
-\end{array}$ & + & $\begin{array}{l}- \\
-\end{array}$ & $\begin{array}{l}- \\
-\end{array}$ & $\begin{array}{l}- \\
-\end{array}$ & $\begin{array}{l}- \\
-\end{array}$ & + & $\begin{array}{l}- \\
-\end{array}$ & $\begin{array}{l}- \\
-\end{array}$ & - & + & $\begin{array}{l}- \\
-\end{array}$ & $\begin{array}{l}- \\
-\end{array}$ & $\begin{array}{l}- \\
-\end{array}$ & - & - & - & - & - & - & - & - & $\begin{array}{l}- \\
-\end{array}$ & - & $\begin{array}{l}- \\
-\end{array}$ & $\begin{array}{l}- \\
-\end{array}$ \\
\hline Monilia sp. & $\begin{array}{l}n \\
b\end{array}$ & - & $\begin{array}{l}+ \\
+\end{array}$ & $\begin{array}{l}- \\
-\end{array}$ & $\begin{array}{l}- \\
-\end{array}$ & $\begin{array}{l}- \\
-\end{array}$ & - & $\begin{array}{l}+ \\
+\end{array}$ & $\begin{array}{l}- \\
-\end{array}$ & $\begin{array}{l}- \\
-\end{array}$ & - & - & $\begin{array}{l}- \\
-\end{array}$ & $\begin{array}{l}- \\
-\end{array}$ & $\begin{array}{l}- \\
-\end{array}$ & - & $\begin{array}{l}- \\
-\end{array}$ & $\begin{array}{l}- \\
-\end{array}$ & $\begin{array}{l}- \\
-\end{array}$ & - & $\begin{array}{l}- \\
-\end{array}$ & - & - & $\begin{array}{l}- \\
-\end{array}$ & $\begin{array}{l}- \\
-\end{array}$ & + & $\begin{array}{l}-1 \\
-\end{array}$ & 1- & - & $\begin{array}{l}- \\
-\end{array}$ & $\begin{array}{l}- \\
-\end{array}$ \\
\hline Mortierella sp. & $\begin{array}{l}\mathrm{n} \\
\mathrm{b}\end{array}$ & - & $\begin{array}{l}- \\
-\end{array}$ & $\begin{array}{l}- \\
-\end{array}$ & $\begin{array}{l}- \\
-\end{array}$ & $\begin{array}{l}- \\
-\end{array}$ & - & $\begin{array}{l}- \\
-\end{array}$ & $\begin{array}{l}- \\
-\end{array}$ & - & - & - & - & + & $\begin{array}{l}- \\
-\end{array}$ & - & $\begin{array}{l}- \\
-\end{array}$ & + & $\begin{array}{l}- \\
-\end{array}$ & - & $\begin{array}{l}. . \\
-\end{array}$ & - & & - & $\begin{array}{l}- \\
-\end{array}$ &. & $\begin{array}{l}- \\
-\end{array}$ & $\begin{array}{l}- \\
-\end{array}$ & - & $\begin{array}{l}- \\
-\end{array}$ & $\begin{array}{l}- \\
-\end{array}$ \\
\hline Mucorales & $\begin{array}{l}n \\
b\end{array}$ & + & $\begin{array}{l}+ \\
-\end{array}$ & $\begin{array}{l}+ \\
-\end{array}$ & $\begin{array}{l}+ \\
-\end{array}$ & $\begin{array}{l}+ \\
-\end{array}$ & - & $\begin{array}{l}+ \\
+\end{array}$ & $\begin{array}{l}+ \\
+\end{array}$ & $\begin{array}{l}+ \\
+\end{array}$ & $\begin{array}{l}+ \\
-\end{array}$ & + & + & $\begin{array}{l}- \\
+\end{array}$ & + & + & $\begin{array}{l}- \\
-\end{array}$ & $\begin{array}{l}- \\
+\end{array}$ & $\begin{array}{l}+ \\
+\end{array}$ & + & + & $\begin{array}{l}+ \\
+\end{array}$ & + & + & $\begin{array}{l}+ \\
+\end{array}$ & - & $\begin{array}{l}- \\
-\end{array}$ & $\begin{array}{l}- \\
-\end{array}$ & + & $\begin{array}{l}- \\
-\end{array}$ & $\begin{array}{l}+ \\
+\end{array}$ \\
\hline Nigrospora sp. & $\begin{array}{l}n \\
b\end{array}$ & - & $\begin{array}{l}- \\
-\end{array}$ & $\begin{array}{l}- \\
-\end{array}$ & - & $\begin{array}{l}- \\
-\end{array}$ & - & $\begin{array}{l}- \\
-\end{array}$ & $\begin{array}{l}- \\
-\end{array}$ & $\begin{array}{l}- \\
-\end{array}$ & $\begin{array}{l}- \\
-\end{array}$ & - & + & $\begin{array}{l}- \\
-\end{array}$ & $\begin{array}{l}- \\
-\end{array}$ & - & - & $\begin{array}{l}- \\
-\end{array}$ & $\begin{array}{l}- \\
-\end{array}$ & - & $\begin{array}{l}- \\
-\end{array}$ & $\begin{array}{l}- \\
-\end{array}$ & - & $\begin{array}{l}- \\
-\end{array}$ & $\begin{array}{l}- \\
-\end{array}$ & - & $\begin{array}{l}- \\
-\end{array}$ & $\begin{array}{l}- \\
-\end{array}$ & - & $\begin{array}{l}- \\
-\end{array}$ & $\overline{-}$ \\
\hline Papularia sp. & $\begin{array}{l}n \\
\mathrm{~b}\end{array}$ & - & $\begin{array}{l}- \\
-\end{array}$ & $\begin{array}{l}- \\
-\end{array}$ & $\begin{array}{l}- \\
-\end{array}$ & $\begin{array}{l}- \\
-\end{array}$ & + & $\begin{array}{l}- \\
-\end{array}$ & $\begin{array}{l}- \\
-\end{array}$ & $\begin{array}{l}- \\
-\end{array}$ & $\begin{array}{l}- \\
-\end{array}$ & + & $\begin{array}{l}- \\
-\end{array}$ & $\begin{array}{l}- \\
-\end{array}$ & $\begin{array}{l}- \\
-\end{array}$ & - & $\begin{array}{l}- \\
-\end{array}$ & $\begin{array}{l}- \\
-\end{array}$ & $\begin{array}{l}- \\
-\end{array}$ & - & - & $\overrightarrow{-}$ & - & $\begin{array}{l}- \\
-\end{array}$ & $\begin{array}{l}- \\
-\end{array}$ & - & $\begin{array}{l}- \\
-\end{array}$ & $\begin{array}{l}- \\
-\end{array}$ & - & $\begin{array}{l}- \\
-\end{array}$ & $\begin{array}{l}- \\
-\end{array}$ \\
\hline Penicillium sp. & $\begin{array}{l}\mathbf{n} \\
\mathrm{t}\end{array}$ & - & $\begin{array}{l}- \\
+\end{array}$ & $\begin{array}{l}+ \\
-\end{array}$ & $\begin{array}{l}- \\
-\end{array}$ & $\begin{array}{l}- \\
+\end{array}$ & + & $\begin{array}{l}+ \\
-\end{array}$ & $\begin{array}{l}+ \\
-\end{array}$ & $\begin{array}{l}+ \\
+\end{array}$ & $\begin{array}{l}- \\
+\end{array}$ & + & $\begin{array}{l}+ \\
+\end{array}$ & + & $\begin{array}{l}+ \\
+\end{array}$ & + & + & $\begin{array}{l}+ \\
-\end{array}$ & $\begin{array}{l}- \\
-\end{array}$ & + & $\begin{array}{l}- \\
-\end{array}$ & $\begin{array}{l}+ \\
-\end{array}$ & + & $\begin{array}{l}- \\
-\end{array}$ & + & - & - & - & + & $\begin{array}{l}- \\
-\end{array}$ & - \\
\hline Peyronellaea sp. & $\begin{array}{l}\mathrm{n} \\
\mathrm{t}\end{array}$ & - & $\begin{array}{l}- \\
-\end{array}$ & - & $\begin{array}{l}- \\
-\end{array}$ & $\begin{array}{l}- \\
-\end{array}$ & - & $\begin{array}{l}- \\
-\end{array}$ & + & $\begin{array}{l}- \\
-\end{array}$ & $\begin{array}{l}- \\
-\end{array}$ & - & $\begin{array}{l}- \\
-\end{array}$ & $\begin{array}{l}- \\
-\end{array}$ & $\begin{array}{l}- \\
-\end{array}$ & - & $\begin{array}{l}- \\
-\end{array}$ & $\begin{array}{l}- \\
-\end{array}$ & $\begin{array}{l}- \\
-\end{array}$ & - & $\begin{array}{l}- \\
-\end{array}$ & $\begin{array}{l}- \\
-\end{array}$ & - & $\begin{array}{l}- \\
-\end{array}$ & $\begin{array}{l}- \\
-\end{array}$ & - & $\begin{array}{l}- \\
-\end{array}$ & - & - & $\begin{array}{l}- \\
-\end{array}$ & - \\
\hline $\begin{array}{l}\text { Pultularia sp. } \\
\text { (Aureobasidium) }\end{array}$ & $\begin{array}{l}\mathrm{n} \\
\mathrm{b}\end{array}$ & - & $\begin{array}{l}- \\
-\end{array}$ & $\begin{array}{l}+ \\
-\end{array}$ & $\begin{array}{l}- \\
-\end{array}$ & $\begin{array}{l}- \\
-\end{array}$ & - & $\begin{array}{l}- \\
-\end{array}$ & $\begin{array}{l}- \\
-\end{array}$ & $\begin{array}{l}- \\
-\end{array}$ & $\begin{array}{l}- \\
-\end{array}$ & - & $\begin{array}{l}- \\
-\end{array}$ & $\begin{array}{l}- \\
-\end{array}$ & $\begin{array}{l}- \\
-\end{array}$ & - & $\begin{array}{l}- \\
-\end{array}$ & $\begin{array}{l}- \\
-\end{array}$ & $\begin{array}{l}+ \\
-\end{array}$ & - & - & $\begin{array}{l}- \\
-\end{array}$ & - & $\begin{array}{l}- \\
-\end{array}$ & $\begin{array}{l}- \\
-\end{array}$ & - & $\begin{array}{l}- \\
-\end{array}$ & $\begin{array}{l}- \\
-\end{array}$ & - & $\begin{array}{l}- \\
-\end{array}$ & $\begin{array}{l}- \\
-\end{array}$ \\
\hline Rhizopus sp. & $\begin{array}{l}\mathrm{n} \\
\mathrm{b}\end{array}$ & - & $\begin{array}{l}- \\
-\end{array}$ & $\begin{array}{l}- \\
-\end{array}$ & $\begin{array}{l}+ \\
-\end{array}$ & $\begin{array}{l}+ \\
-\end{array}$ & - & $\begin{array}{l}+ \\
+\end{array}$ & + & $\begin{array}{l}- \\
-\end{array}$ & $\begin{array}{l}+ \\
-\end{array}$ & - & $\begin{array}{l}- \\
-\end{array}$ & $\begin{array}{l}- \\
-\end{array}$ & + & - & $\begin{array}{l}- \\
-\end{array}$ & $\begin{array}{l}+ \\
-\end{array}$ & - & + & $\begin{array}{l}- \\
+\end{array}$ & - & + & $\overline{+}$ & + & - & + & $\overline{+}$ & - & $\overline{-}$ & $\overline{+}$ \\
\hline Stachybotrys sp. & $\begin{array}{l}n \\
\mathrm{t}\end{array}$ & - & $\overline{-}$ & - & - & $\begin{array}{l}- \\
-\end{array}$ & - & $\begin{array}{l}- \\
-\end{array}$ & $\begin{array}{l}- \\
\cdots\end{array}$ & - & - & - & $\begin{array}{l}+ \\
-\end{array}$ & $\begin{array}{l}- \\
-\end{array}$ & $\begin{array}{l}- \\
-\end{array}$ & - & $\begin{array}{l}- \\
-\end{array}$ & $\begin{array}{l}- \\
-\end{array}$ & $\begin{array}{l}- \\
-\end{array}$ & - & - & - & - & - & - & $\dot{-}$ & - & - & - & - & - \\
\hline Stemphylium sp. & $\begin{array}{l}n \\
b\end{array}$ & - & $\begin{array}{l}- \\
-\end{array}$ & - & $\begin{array}{l}- \\
-\end{array}$ & $\begin{array}{l}- \\
-\end{array}$ & - & - & - & $\overrightarrow{-}$ & - & - & - & $\begin{array}{l}- \\
-\end{array}$ & $\begin{array}{l}- \\
-\end{array}$ & - & $\begin{array}{l}+ \\
-\end{array}$ & - & - & - & - & $\begin{array}{l}- \\
-\end{array}$ & - & $\begin{array}{l}- \\
-\end{array}$ & - & - & $\begin{array}{l}- \\
-\end{array}$ & $\begin{array}{l}- \\
-\end{array}$ & - & $\begin{array}{l}- \\
-\end{array}$ & - \\
\hline Thielaviopsis $\mathrm{sp}$. & $\begin{array}{l}n \\
b\end{array}$ & - & $\begin{array}{l}- \\
-\end{array}$ & $\begin{array}{l}- \\
-\end{array}$ & $\begin{array}{l}- \\
-\end{array}$ & - & - & $\begin{array}{l}- \\
-\end{array}$ & $\begin{array}{l}- \\
\cdots\end{array}$ & - & $\begin{array}{l}- \\
-\end{array}$ & - & + & $\begin{array}{l}- \\
-\end{array}$ & $\begin{array}{l}- \\
-\end{array}$ & - & $\begin{array}{l}- \\
-\end{array}$ & $\begin{array}{l}- \\
-\end{array}$ & $\begin{array}{l}- \\
-\end{array}$ & - & $\begin{array}{l}- \\
-\end{array}$ & - & & - & $\begin{array}{l}- \\
-\end{array}$ & - & $\begin{array}{l}- \\
-\end{array}$ & $\begin{array}{l}- \\
-\end{array}$ & - & - & - \\
\hline Trichoderma sp. & $\begin{array}{l}n \\
b\end{array}$ & - & $\begin{array}{l}- \\
-\end{array}$ & $\begin{array}{l}+ \\
-\end{array}$ & $\begin{array}{l}+ \\
-\end{array}$ & $\begin{array}{l}+ \\
-\end{array}$ & + & $\begin{array}{l}+ \\
+\end{array}$ & $\begin{array}{l}+ \\
-\end{array}$ & $\begin{array}{l}- \\
-\end{array}$ & $\begin{array}{l}- \\
-\end{array}$ & + & $\begin{array}{l}+ \\
-\end{array}$ & $\begin{array}{l}+ \\
-\end{array}$ & $\begin{array}{l}+ \\
-\end{array}$ & + & $\begin{array}{l}+ \\
-\end{array}$ & + & $\begin{array}{l}- \\
-\end{array}$ & + & $\begin{array}{l}- \\
-\end{array}$ & - & + & $\begin{array}{l}- \\
-\end{array}$ & $\begin{array}{l}- \\
-\end{array}$ & + & - & - & - & - & - \\
\hline Verticillium sp. & $\begin{array}{l}n \\
b\end{array}$ & - & $\begin{array}{l}- \\
-\end{array}$ & $\begin{array}{l}- \\
-\end{array}$ & $\begin{array}{l}- \\
-\end{array}$ & $\begin{array}{l}- \\
-\end{array}$ & - & $\begin{array}{l}- \\
-\end{array}$ & $\begin{array}{l}+ \\
-\end{array}$ & - & $\begin{array}{l}- \\
-\end{array}$ & - & $\begin{array}{l}- \\
-\end{array}$ & $\begin{array}{l}+ \\
-\end{array}$ & $\begin{array}{l}- \\
-\end{array}$ & - & $\begin{array}{l}- \\
-\end{array}$ & $\begin{array}{l}- \\
-\end{array}$ & $\begin{array}{l}- \\
-\end{array}$ & - & $\begin{array}{l}- \\
+\end{array}$ & $\begin{array}{l}- \\
-\end{array}$ & - & $\begin{array}{l}- \\
-\end{array}$ & $\begin{array}{l}- \\
-\end{array}$ & - & - & - & - & $\begin{array}{l}- \\
-\end{array}$ & $\begin{array}{l}- \\
-\end{array}$ \\
\hline $\begin{array}{l}\text { Champignons } \\
\text { non déterminés }\end{array}$ & $\begin{array}{l}n \\
\mathrm{~b}\end{array}$ & - & $\begin{array}{l}- \\
-\end{array}$ & $\begin{array}{l}- \\
-\end{array}$ & $\begin{array}{l}- \\
-\end{array}$ & $\begin{array}{l}- \\
-\end{array}$ & + & $\begin{array}{l}- \\
-\end{array}$ & $\begin{array}{l}- \\
-\end{array}$ & $\begin{array}{l}- \\
-\end{array}$ & $\begin{array}{l}- \\
-\end{array}$ & - & $\begin{array}{l}- \\
-\end{array}$ & $\begin{array}{l}- \\
-\end{array}$ & $\begin{array}{l}- \\
-\end{array}$ & + & $\begin{array}{l}- \\
-\end{array}$ & $\begin{array}{l}- \\
-\end{array}$ & - & - & + & $\begin{array}{l}- \\
-\end{array}$ & - & $\begin{array}{l}- \\
+\end{array}$ & $\begin{array}{l}- \\
-\end{array}$ & - & $\begin{array}{l}- \\
-\end{array}$ & $\begin{array}{l}- \\
-\end{array}$ & + & $\begin{array}{l}+ \\
+\end{array}$ & - \\
\hline $\begin{array}{l}\text { Nb. genres par } \\
\text { prélèvement } \\
\text { (y compris bact. } \\
\text { et actino). }\end{array}$ & $n$ & 8 & $\begin{array}{l}3 \\
4\end{array}$ & $\begin{array}{r}11 \\
1\end{array}$ & $\begin{array}{l}7 \\
2\end{array}$ & $\begin{array}{l}7 \\
2\end{array}$ & 11 & $\begin{array}{l}9 \\
8\end{array}$ & $\begin{array}{c}12 \\
5\end{array}$ & $\begin{array}{l}6 \\
4\end{array}$ & $\begin{array}{l}6 \\
3\end{array}$ & 10 & $\begin{array}{r}11 \\
5\end{array}$ & $\begin{array}{l}8 \\
4\end{array}$ & $\begin{array}{l}8 \\
4\end{array}$ & 12 & $\begin{array}{l}7 \\
4\end{array}$ & $\begin{array}{l}8 \\
5\end{array}$ & $\begin{array}{l}6 \\
4\end{array}$ & 6 & $\begin{array}{l}6 \\
8\end{array}$ & $\begin{array}{l}7 \\
4\end{array}$ & 7 & $\begin{array}{l}4 \\
6\end{array}$ & $\begin{array}{l}7 \\
5\end{array}$ & 4 & $\begin{array}{l}5 \\
4\end{array}$ & $\begin{array}{l}4 \\
6\end{array}$ & 6 & $\begin{array}{l}5 \\
4\end{array}$ & $\begin{array}{l}6 \\
5\end{array}$ \\
\hline
\end{tabular}

d'installation) sont assurées 4 années sur 5 dans notre région méditerranéenne.

Son efficacité est améliorée par l'emploi de films plus performants du type E.V.A. ou de plastiques alvéolés.

Dans de bonnes conditions, on obtient une réduction très importante de la nématofaune et de la mycoflore sur au moins $40 \mathrm{~cm}$ de profondeur et une action sur la germination des semences de mauvaises herbes. Par contre, les populations bactériennes ne sont que peu ou pas affectées.
Des essais nous paraissent indispensables dans d'autres types de sols pour vérifier si la répétition de l'application du procédé ne se traduit pas par la sélection d'organismes moins affectés par la chaleur, susceptibles de remettre en cause les effets éradicants de l'élévation modérée de température obtenue par la solarisation. 


\section{RÉFÉRENCES BIBLIOGRAPHIQUES}

Demeure Y., 1978. Les causes de la survie de certains nématodes phytoparasites (Scutellonema cavenessi et Meloidogyne sp.) pendant la saison sèche dans le Sahel sénégalais. Thèse $3^{\text {e }}$ cycle, Univ. $\mathrm{Cl}$. Bernard, Lyon, $105 \mathrm{p}$.

Garibaldi A., 1983. Attempts to use soil solarisation in closed greenhouses in northern Italy for the control of soil-borne pathogens of tomato. 2nd Int. Symp. Soil Disinfect., Leuven (Belgium), 46 .

Goisque M. J., Louvet H., Martin C., Lagier J., Davet P., Couteaudier Y., Louvet J., 1984. La désinfection solaire du sol. Un moyen de lutte contre la maladie des racines liègeuses de la tomate. Rev. Hortic., P.H.M., 247, 49-53.

Henin S., 1977. Cours de physique du sol, ORSTOM. Ed. Tome 1, $159 \mathrm{p}$. Tome 2, $222 \mathrm{p}$.
Johnson A. W., Jaworski C. A., Glaze N. C., Summer D. R., Chalfant R. B., 1981. Effects of film mulch and soil pesticides on nematodes, weeds and yield of vegetable crops. J. Nematol., 13, 141-148.

Katan J., Greenberger A., Alan H., Grinstein H., 1976. Solar heating by polyethylene mulching for the control of diseases caused by soil-borne pathogens. Phytopathology, 66, 683-688.

Malathrakis N. E., Kapetanakis G. E., Linardakis, 1983. Brown root rot of tomato and its control in Crete. Ann. Appl. Biol., 102, 251-256.

Osman A. R., Sahaby A. F., 1983. Control of Rhizoctonia solani by soil solarisation: 2nd Int. Symp. Soil Disinf., Leuven (Belgium). Abstract, 47.

Tjamos E. C., Faridis A., 1980. Control of soil-borne pathogens by solar heating in plastic houses. Proc. Sth Cong. mediterr. Phytopathol. Union, Patras, Greece, 82-84. 